\title{
A Panel of Urinary Long Non-coding RNAs Differentiate Bladder Cancer from Urocystitis
}

\author{
Xiao $\mathrm{Yu}^{1^{*}}$, Ruiwei Wang ${ }^{2 *}$, Chenglin Han ${ }^{1}$, Zilong Wang1, Xunbo Jin ${ }^{1 凶}$ \\ 1. Department of Urology, Shandong Provincial Hospital Affiliated to Shandong University, No. 324 Jingwu Road, Huaiyin District, Jinan, Shandong, 250021, \\ China. \\ 2. Department of Anesthesiology, Shandong Provincial Hospital Affiliated to Shandong University, No. 324 Jingwu Road, Huaiyin District, Jinan, Shandong, \\ 250021, China. \\ *Both authors contributed equally to this work. \\ $\bowtie$ Corresponding author: Dr. Xunbo Jin, Email: jxb@sdu.edu.cn \\ (C) The author(s). This is an open access article distributed under the terms of the Creative Commons Attribution License (https://creativecommons.org/licenses/by/4.0/). \\ See http://ivyspring.com/terms for full terms and conditions.
}

Received: 2019.05.26; Accepted: 2019.09.27; Published: 2020.01.01

\begin{abstract}
Liquid biopsy is becoming a promising method for non-invasive cancer detection. In several proof-of-concept studies, long non-coding RNAs (IncRNAs) were found to be potential biomarkers for bladder cancer detection. The objective of this study was to discover a panel of cell-free, urinary IncRNAs as liquid biopsy biomarkers to non-invasively differentiate bladder cancer from chronic urocystitis. To this end, we collected urine samples from both bladder cancer patients and urocystitis patients. These samples were divided into discovery group and validation group. In the discovery group, the expression levels of 16 cell-free urinary IncRNAs were measured by QPCR to discover candidate biomarkers. The diagnostic performance of the candidate IncRNA biomarkers was then evaluated, which led to a panel of IncRNA biomarkers for bladder cancer detection. The performance of this panel of biomarkers was further evaluated in the validation group to see if these IncRNA biomarkers could discriminate the bladder cancer patients from urocystitis patients. We found that all of the 16 IncRNAs evaluated in this study demonstrated significant difference $(p<0.05)$ of expression between bladder cancer patients and urocystitis patients. Nine IncRNAs provided decent diagnostic performance with area under the receiver operating characteristic (ROC) curve (AUC) reaching 0.70 or higher. We then selected the top four IncRNAs, namely UCA1-201, HOTAIR, HYMAI and MALAT1, to form a panel of urinary biomarkers. Using this panel, bladder cancer patients could be discriminated from urocystitis patients, with sensitivity and specificity reaching $95.7 \%$ and $94.3 \%$, respectively. Finally, we confirmed the applicability of the four-IncRNA panel in an independent validation study that included 60 bladder cancer patients and 60 urocystitis patients. Our study paves the way for further studies aimed at large-scale clinical tests of developing IncRNA biomarkers in urine for bladder cancer diagnostics.
\end{abstract}

Key words: Biomarker, NMIBC, urocystitis, non-coding RNA, expression.

\section{Introduction}

Malignant bladder carcinomas are the most common tumors in urinary systems. Bladder cancer concerns approximately 549,393 new cases each year worldwide and its incidence is constantly increasing $[1,2]$. At time of diagnosis, non-muscle invasive bladder cancer (NMIBC) represents the majority of cases, accounting for 70\% [1-9]. Early diagnosis and treatment of cancerous or precancerous lesions is thought to be important for reducing the risk of relapse and improving the prognosis of NMIBC [10].
However, the differentiation of chronic urocystitis from NMIBC can be particularly difficult, especially after previous treatment with attenuated mycobacteria (Bacillus Calmette-Guèrin) for deliberate induction of an inflammatory reaction [6, 8]. This treatment is often a primary treatment option for NMIBC aside from early cystectomy [6, 8]. Therefore, correct discrimination of urocystitis from NMIBC calls for a panel of biomarkers with both high sensitivity and high specificity. 
Recently, several reports have highlighted the role of long non-coding RNAs (lncRNAs) as urine-based biomarkers for diagnosis of bladder cancer [1, 10-22]. Long non-coding RNAs are transcripts longer than 200 nucleotides that are not translated into protein [23]. LncRNAs do not contain open reading frames, but have conservative secondary structures. LncRNAs could interact with DNA, RNA or proteins as molecular sponges, scaffolds and activators to play important regulatory roles in a variety of biological processes [11]. For example, Wang and collaborators [13] determined that high expression of UCA1 (Urothelial cancer associated 1) in urine sediments allows detection of high-grade superficial bladder tumors. Another study [14] showed that overexpression of several lncRNAs, such as HOTAIR, HOX-AS-2, MALAT1, HYMAI, LINC00477, LOC100506688 and OTX2-AS1, has been found in urine exosomes of high-grade MIBC patients. Several other studies [10,12] also proposed various panels of lncRNAs as liquid biopsy biomarkers to discriminate bladder cancer patients from healthy controls.

Encouraged by these successes, in this study, we attempted to discover and evaluate the prediction power of a panel of lncRNAs biomarkers to discriminate bladder cancer from urocystitis. We chose totally sixteen lncRNAs as our candidate biomarkers, as suggested by previous studies $[1$, 10-22]. We conducted a study of 140 NMIBC patients and 140 urocystitis patients. We found that the expression profiles of sixteen cell-free lncRNAs evaluated in urine were significantly different between NMIBC patients and urocystitis patients. Among these biomarkers, we chose four lncRNAs, namely UCA1-201, HOTAIR, HYMA1 and MALAT1, as our panel of lncRNA biomarkers for further investigation, due to their high value of area under curve (AUC) of the receiver-operating characteristic (ROC) curve. We found that this panel discriminated the NMIBC patients from urocystitis patients with high sensitivity (95.7\%) and high specificity (94.3\%). To further evaluate the clinical performance of our method, we then applied this cell-free, urinary lncRNA panel to an independent clinic study that included 60 NMIBC patients and 60 urocystitis patients. The specificity of our method remained as high as $96.7 \%$ : only 2 out of 60 urocystitis patients scored as cancer positive. The sensitivity of our method was $93.3 \%$ : 56 out of 60 cancer patients scored as cancer positive. These studies confirmed that the lncRNA panel could serve as a robust, accurate, and non-invasive method for bladder cancer detection. To our best knowledge, our study represents the first attempt of using cell-free urinary lncRNA biomarkers for non-invasive differentiation of bladder cancer from urocystitis.

\section{Materials and methods}

\section{Patient populations and ethnics}

We were approved for this study by the Institutional Review Board of Shandong Provincial Hospital Affiliated to Shandong University (Jinan, China) and conducted this study in accordance with the principles of Declaration of Helsinki. For all research participants, we obtained written-informed consent forms. In the cohort for biomarker discovery phase (Table 1), a total of 140 NMIBC patients, and 140 people that were diagnosed as severe urocystitis with reactive urothelial atypia (RUA) were recruited in the period of April 19th, 2015 and January 17th 2018. The following criteria were used to include or exclude research participants: 1) inclusion criteria: confirmed bladder cancer that was diagnosed via cytologic examination; and 2) exclusion criteria: NMIBC patients that had chemotherapy/radiotherapy within 1 month, or NMIBC patients that were diagnosed of other malignancies within five years prior to urine collection.

Table 1. Demographic Information of All Subjects Used in This Study.

\begin{tabular}{|c|c|c|c|}
\hline Groups & Training datasets & Validation datasets & $P$-Value \\
\hline \multirow[t]{14}{*}{ Control Group } & $\begin{array}{l}\text { Patients recruited in } \\
\text { training phase }\end{array}$ & $\begin{array}{l}\text { Patients recruited in } \\
\text { validation phase }\end{array}$ & \\
\hline & 140 urocystitis patients & 60 urocystitis patients & \\
\hline & Age (years) & Age (years) & 0.58 \\
\hline & $<66: 74(52.85 \%)$ & $<66: 29(48.33 \%)$ & \\
\hline & $\geq 66: 66(47.15 \%)$ & $\geq 66: 31(51.67 \%)$ & \\
\hline & Cigarette smokers & Cigarette smokers & 0.32 \\
\hline & $37(26.43 \%)$ & $17(28.3 \%)$ & \\
\hline & Alcohol drinkers & Alcohol drinkers & 0.24 \\
\hline & $19(13.57 \%)$ & $8(13.33 \%)$ & \\
\hline & Sex & Sex & 0.34 \\
\hline & Male: $92(65.71 \%)$ & Male: $39(65.00 \%)$ & \\
\hline & Female: 48 (34.29\%) & Female: $21(35.00 \%)$ & \\
\hline & Condition & Condition & 0.89 \\
\hline & Cystitis: 140 (100\%) & Cystitis: 60 (100\%) & \\
\hline \multirow[t]{15}{*}{$\begin{array}{l}\text { Bladder Cancer } \\
\text { Group }\end{array}$} & $\begin{array}{l}\text { Patients recruited in } \\
\text { training phase }\end{array}$ & $\begin{array}{l}\text { Patients recruited in } \\
\text { validation phase }\end{array}$ & \\
\hline & 140 NMIBC patients & 60 NMIBC patients & \\
\hline & Age (years) & Age (years) & 0.52 \\
\hline & $<66: 72(51.43 \%)$ & $<66: 33(55.00 \%)$ & \\
\hline & $\geq 66: 68(48.57 \%)$ & $\geq 66: 27(45.00 \%)$ & \\
\hline & Cigarette smokers & Cigarette smokers & 0.31 \\
\hline & $45(32.14 \%)$ & $19(31.7 \%)$ & \\
\hline & Alcohol drinkers & Alcohol drinkers & 0.27 \\
\hline & $23(16.43 \%)$ & $9(15.00 \%)$ & \\
\hline & Sex & Sex & 0.93 \\
\hline & Male: 96 (68.57\%) & Male: $41(68.33 \%)$ & \\
\hline & Female: $44(31.43 \%)$ & Female: 19 (31.67\%) & \\
\hline & Tumor stage & Tumor stage & 0.21 \\
\hline & Ta-T1: 78 (55.71\%) & Ta-T1: 35 (58.33\%) & \\
\hline & T2-T4: $62(44.29 \%)$ & T2-T4: 25 (41.67\%) & \\
\hline
\end{tabular}




\section{Urine sample collection and measurement of IncRNA expression levels in urine}

Urine samples were collected on the day before treatment and kept on ice during collection. To remove cells and debris, urine samples were centrifuged at 1,500 $\mathrm{g}$ for 10 minutes and $13,800 \mathrm{~g}$ for 15 minutes at $4^{\circ} \mathrm{C}$ by following a previously published protocol [10]. We followed a protocol that was previously published for extracting cell-free, urinary lncRNA [10]. In brief, total RNA was isolated from 400 $\mu \mathrm{L}$ urine by using the miRNeasy Mini Kit (Qiagen) and following the manufacturer's instructions. We used NanoDrop spectrophotometer to measure the concentration of isolated RNA. Reverse transcription of urinary IncRNA was conducted by using the PrimeScript $^{\mathrm{TM}}$ RT reagent kit (Takara, Dalian, Liaoning), in which the $20 \mu \mathrm{L} \mathrm{RT}$ volume contained 1 $\mu \mathrm{g}$ of template RNA, $4 \mu \mathrm{L}$ of $5 \times$ PrimeScript Buffer, 1 $\mu \mathrm{L}$ of PrimeScript RT Enzyme Mix I, $1 \mu \mathrm{L}$ of Oligo dT Primer and RNase - free $\mathrm{dH} 2 \mathrm{O}$. The mixture was briefly centrifuged and incubated at $37^{\circ} \mathrm{C}$ for 30 minutes, followed by incubation at $85^{\circ} \mathrm{C}$ for 5 seconds and $4^{\circ} \mathrm{C}$ for 60 minutes. The quantitative polymerase chain reaction was conducted in a $25 \mu \mathrm{L}$ reaction system, which contained $12.5 \mu \mathrm{L}$ of SYBR® Premix Ex $\mathrm{Taq}^{\mathrm{TM}}, 0.5 \mu \mathrm{L}$ of ROX Reference Dye $\alpha, 1 \mu \mathrm{L}$ of forward primer $(10 \mu \mathrm{mol} / \mathrm{L}), 1 \mu \mathrm{L}$ of reverse primer, 8 $\mu \mathrm{L}$ of RNas - free $\mathrm{dH} 2 \mathrm{O}$ and $2 \mu \mathrm{L}$ of cDNA on a CFX-96 real-time PCR System using the SYBR® Premix Ex Taq ${ }^{\mathrm{TM}}$ (Takara, Dalian, Liaoning). All reactions were performed in triplicate. GAPDH was used as an internal control. Raw $\mathrm{Ct}$ data was normalized by subtracting GAPDH $\mathrm{Ct}$ values from the biomarker $\mathrm{Ct}$ values to generate $\Delta \mathrm{Ct}$. The relative gene expression were calculated by following the $2^{-\Delta C T}$ method as reported previously [24], where relative gene expression in a urine sample $=2 \wedge(-\Delta \mathrm{Ct})$ and multiplying by 1000 .

\section{Statistical methods and machine learning analysis}

Boxplot of relative lncRNA expression level was conducted by using MedCalc software (MedCalc Software bv, Ostend, Belgium). Two-sided p-value was calculated when comparing the expression level between patient group and control group. A p-value smaller than 0.05 was chosen as statistically significant. For each biomarker, we performed receiver operating characteristic (ROC) analysis, followed by calculating the area under the curve (AUC) using MedCalc software. Based on the AUC value of each biomarker, we could decide whether or not a biomarker was discriminative in separating NMIBC patients and urocystitis patients. We chose the biomarkers that had AUC values larger than 0.70 to develop a predictive model. We selected decision tree algorithm as our classifier and applied 10-fold cross-validations to avoid overfitting. The classification was conducted by using the data collected in the biomarker discovery phase. Python scikit-learn package was used for the computational analysis. The classifier was then used for the data collected in the validation phase to predict whether or not a sample was from NMIBC patients.

\section{Results}

\section{Study design}

We designed our study by including a biomarker discovery phase and an independent clinical validation phase. The discovery phase aimed at designing a panel of cell-free urinary lncRNA biomarkers to differentiate NMIBC patients from urocystitis patients. The validation phase aimed at using this panel in clinical diagnosis to evaluate its performance of NMIBC detection. In discovery phase, we recruited 140 NMIBC patients and 140 urocystitis patients (Table 1) and collected urine samples to measure the cell-free expression level of sixteen candidate lncRNAs. These data formed the training datasets, on which we applied a machine learning model and 10-fold cross-validation to determine the panel's sensitivity and specificity. Next, using the developed multi-lncRNA panel, we performed a blinded test to predict if a urine sample was from a NMIBC patient or urocystitis patient. We recruited a total of 60 NMIBC patients and 60 urocystitis patients in the validation phase.

\section{Evaluating expression profiles of candidate IncRNA biomarkers in urine for NMIBC detection}

We analyzed the expression levels of totally sixteen candidate biomarkers in two groups of the training datasets: a NMIBC group and urocystitis group (Figure 1). The NMIBC cancer group consisted of 140 NMIBC patients, while the urocystitis group consisted of 140 patients diagnosed with severe urocystitis with RUA. These candidate lncRNA biomarkers were selected because they have been previously reported to be able to discriminate bladder cancer patients from healthy controls $[10,12,14]$. We compared the extracellular expression levels of each candidate biomarker in urine between NMIBC group and urocystitis group. We found that all of the candidate biomarkers demonstrated elevated expression levels in the NMIBC group except for UCA1-201 and GAS5, with HYMA1 showing the largest up-regulation (1.31-fold). UCA1-201 and GAS5, on the other hand, demonstrated decreased 
expression level in the NMIBC group (1.48-fold and 1.18-fold, respectively). The differentiated expressions were significant $(p<0.05)$ for all candidate biomarkers, indicating the feasibility of using these biomarkers for potential classification of bladder cancer.
LINC00355

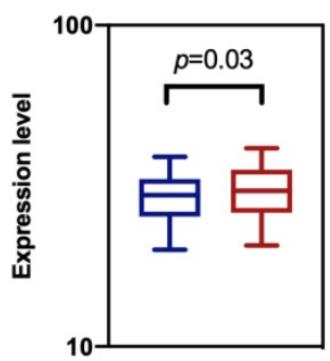

MTND5

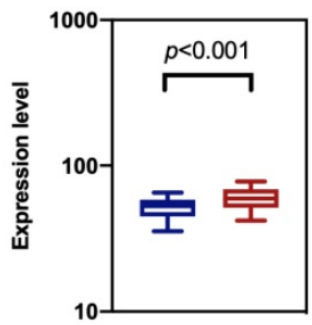

ANRIL

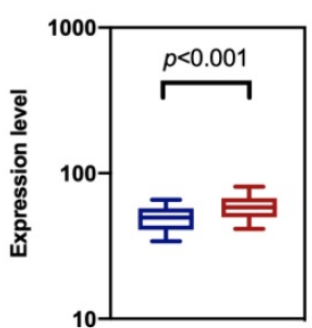

UCA1-203

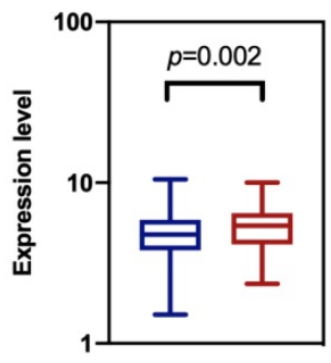

GAS5

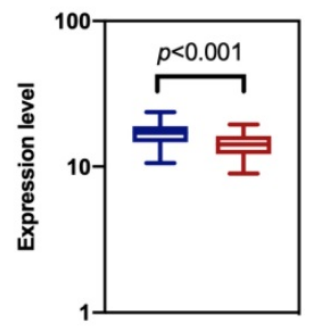

HOTAIR

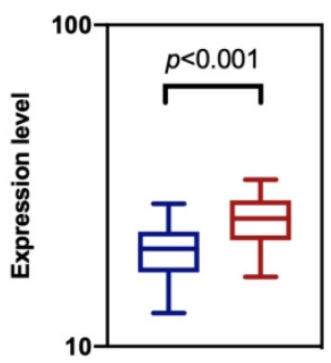

HOTAIR

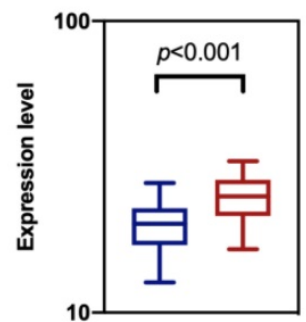

UCA1-201

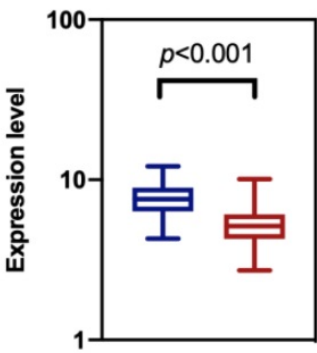

HOX-AS-2

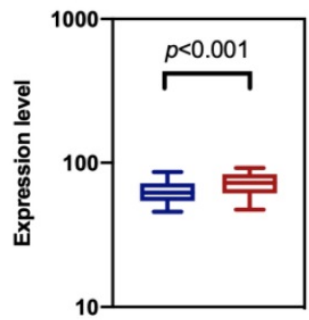

HYMA1

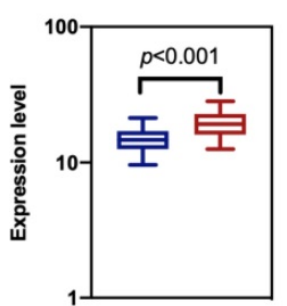

linc_ROR

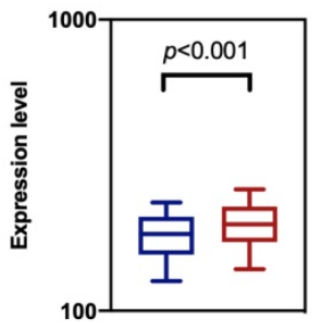

OCT4

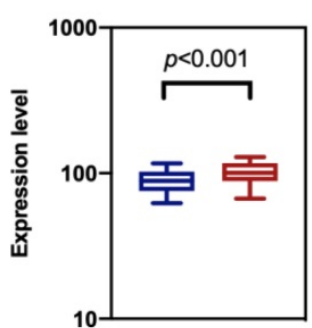

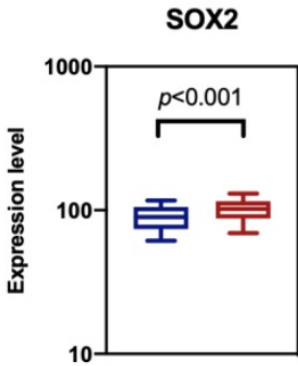

Figure 1. Comparison of cell-free expression levels of sixteen candidate IncRNA biomarkers in urine between NMIBC group (blue) and urocystitis group (red). The $p$-value from t-test analysis was calculated for each comparison. We considered $p<0.05$ as statistically significant. 


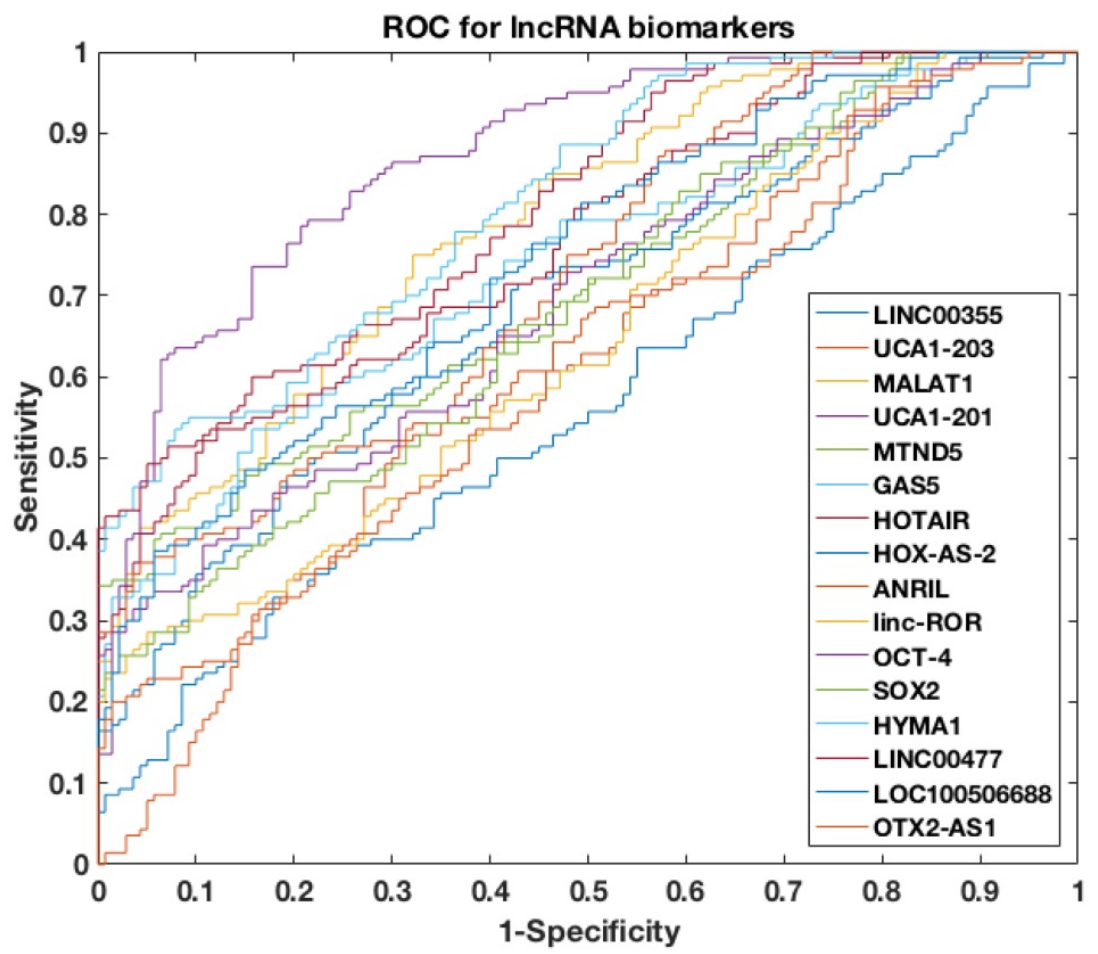

Figure 2. ROC curves of single IncRNA biomarker used to differentiate NMIBC and urocystitis in the training datasets.

\section{Discovering a panel of cell-free urinary IncRNA biomarkers}

To further assess the power of differentiating NMIBC patients from urocystitis patients, the receiver operating characteristic (ROC) curve was evaluated for each candidate biomarker, followed by area under curve (AUC) calculation (Figure 2). Among the sixteen candidate biomarkers, nine biomarkers (i.e., UCA1-201, HOTAIR, HYMA1, MALAT1, LINC00477, LOC100506688, GAS5, ANRIL, and MTND5) demonstrated AUC values higher than 0.70 (i.e., an AUC value that normally suggests decent separation of clinical positives from negatives), while the other seven biomarkers had AUC values below 0.70. The AUC of top four biomarkers, i.e., UCA1-201, HOTAIR, HYMA1 and MALAT1, were even higher than 0.80 , suggesting superior diagnostic performance in differentiating NMIBC and urocystitis.

To further explore the potential of using cell-free urinary lncRNAs as biomarkers to differentiate NMIBC and urocystitis, we then constructed a four-lncRNA panel that included UCA1-201, HOTAIR, HYMA1 and MALAT1. We applied a machine-learning model to predict NMIBC occurrence using the four-lncRNA panel. The input of this predictive model was the cell-free expression levels of the four lncRNAs, paired with the phenotype data (i.e., NMIBC or urocystitis). Totally 280 datasets were retrieved in the training phase, which was then used to train the model. Decision-tree algorithm was chosen as the classifier and python scikit-learn package was adopted for computation, which automatically adjusted the nodes and connections of the decision tree to optimize the fitting [25]. The predictive dramatically improved the discriminative power, as the AUC of the ROC curve reached 0.95.

\section{Validating biomarker panel with independent datasets}

To further test if our four-lncRNA panel could be generally applied for differentiating NMIBC patients from urocystitis patients, we sought validation in independent datasets. In this phase of independent biomarker panel validation, we conducted a study of 60 NMIBC patients and 60 urocystitis patients, blinded. As shown in Table 1, demographic information of the participants in the validation phase was similar to those in the discovery phase. The urine samples were obtained from participants in the validation phase, blinded, and analyzed for the cell-free expression level of UCA1-201, HOTAIR, HYMA1 and MALAT1. Compared to samples collected from NMIBC patients and urocystitis patients in the discovery phase, none of the lncRNA expression showed significant difference $(p>0.05)$ in the validation phase. Using the machine learning guided, predictive model trained in the discovery phase, we made predictions on the samples in the validation phase (i.e., whether or not one sample was from a NMIBC patient). Totally 58 out of 60 urocystitis patients scored negative, and 56 of 60 
NMIBC patients scored positive (Figure 3). The sensitivity reached $93.3 \%$ and the specificity $96.7 \%$. The high sensitivity and specificity supported the application of the four-lncRNA panel in urine samples as a potentially non-invasive approach for clinical diganosis for NMIBC.

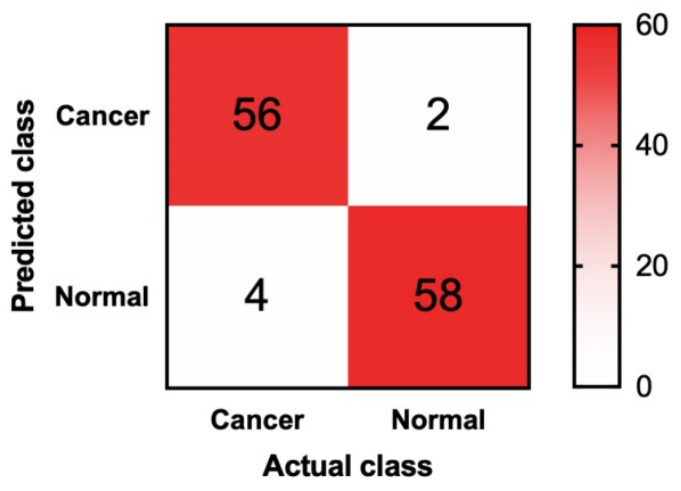

$$
\begin{aligned}
& \text { Sensitivity }=93.3 \% \\
& \text { Specificity }=96.7 \%
\end{aligned}
$$

Figure 3. Confusion matrix of applying the four-IncRNA panel to differentiate NMIBC and urocystitis in the validation datasets.

\section{Discussion}

Our discovery of a panel of four lncRNA biomarkers in urine (i.e., UCA1-201, HOTAIR, HYMA1 and MALAT1) presents a promising and unique means for differentiating NMIBC patients from urocystitis patients. Using this panel, we were able to achieve high specificity and high sensitivity in both training datasets and independent validation datasets. The candidate IncRNA biomarkers have been evaluated on differentiating bladder cancer patients from healthy controls $[10,12,14]$. Our study complements previous efforts and expand the usage of liquid biopsy lncRNA biomarkers for discriminating bladder cancer patients from inflammatory patients. To our best knowledge, it is the first time that cell-free urinary lncRNA biomarkers were used to non-invasively separate bladder cancer patients and urocystitis patients.

One of the novel findings from this study is that certain lncRNA biomarkers, which were used to detect bladder tumors from healthy controls, did not perform well in separating bladder tumors from urocystitis. In this study, we evaluated sixteen lncRNA biomarkers, all of which were found to be effective in discriminating bladder tumors from healthy controls [10, 12-14]. However, among the sixteen biomarkers, seven of them, namely LINC00355, UCA1-203, HOX-AS-2, Linc-ROR, OCT4, SOX2 and OTX2-AS1, did not demonstrate sufficient discriminatory power to separate bladder tumors from urocystitis, as indicated by their low AUC values $(\mathrm{AUC}<0.70)$. While investigating the biomolecular mechanism of lncRNA biomarkers is beyond the scope of this study, we do want to raise the hypothesis that during the development of urocystitis, the transcriptome of urine was altered by inflammation, which could potentially cause the reduced effectiveness of certain biomarkers. Our finding also highlights the necessity of developing "cancer vs. inflammation" biomarkers to specifically discriminate bladder tumors from urocystitis in clinical applications.

\begin{tabular}{|c|c|}
\hline Primer name & Sequence \\
\hline LINC00355_F & TGGGTCTCCTCTGAGCTGTT \\
\hline LINC00355_R & TGTCCTGTGTCCAGGATGAA \\
\hline UCA1-201_F & GCTTAGTGGCTGAAGACTGATG \\
\hline UCA1-201_R & TCATATGGCTGGGAATCCTC \\
\hline UCA1-203_F & GCATCCAGGACAACACAAAG \\
\hline UCA1-203_R & ACССTTTTCCCATAGGTGTG \\
\hline MALAT1_F & СTTCCCTAGGGGATTTCAGG \\
\hline MALAT1_R & GCCCACAGGAACAAGTCCTA \\
\hline HOTAIR_F & TCCCCTACTGCAGGCTTCTA \\
\hline HOTAIR_R & CCTAATATCCCGGAGGTGGCT \\
\hline HOXA-AS2_F & GСTCTCTCCTGCCTTCCTG \\
\hline HOXA-AS2_R & AGCTTGGCCTACTGTGGAAA \\
\hline ANRIL_F & GCCTCATTCTGATTCAACAGC \\
\hline ANRIL_R & GATCTCCCCGGTTTTCTTCT \\
\hline Linc-RoR_F & CTGGCTTTCTGGTTTGACG \\
\hline Linc-RoR_R & CAGGAGGTTACTGGACTTGGAG \\
\hline OCT4_F & GAAAGCGAACCAGTATCGAGAAC \\
\hline OCT4_R & CСССТGAGAAAGGAGACCCA \\
\hline SOX2_F & ACCAGCTCGCAGACCTACAT \\
\hline SOX2_R & TGGAGTGGGAGGAAGAGGTA \\
\hline HYMA1_F & TTGCCTTTGTTTTCCTCCAG \\
\hline HYMA1_R & ACGCAATTGAATGGGAAAG \\
\hline LINC00477_F & СТССТССТССТTGGССTACT \\
\hline LINC00477_R & CAGCCTGGACAACAGAGTGA \\
\hline LOC100506688_F & GTGGCTGAGAGGCTACAGGA \\
\hline LOC100506688_R & GAGGTCTGTCGCTGGACTTT \\
\hline OTX2-AS1_F & TGAAAGCGATGATGATGTCG \\
\hline OTX2-AS1_R & GCAACAAGAGCCAGGTAAGG \\
\hline MTND5_F & TCATAATAGTTACAATCGGCAT \\
\hline MTND5_R & TAATGAGAAATCCTGCGAATA \\
\hline GAS5_F & CTTCTGGGCTCAAGTGATCCT \\
\hline GAS5_R & TTGTGCCATGAGACTCCATCAG \\
\hline GAPDH_F & CTGGGCTACACTGAGCACC \\
\hline GAPDH_R & AAGTGGTCGTTGAGGGCAATG \\
\hline
\end{tabular}

Table 2. Primers Used in This Study.

We also would like to pinpoint a few limitations of this study. As mentioned in the paragraph above, in this study we aimed at discovering and validating biomarkers of bladder tumors, but not focused on uncovering the molecular insights into the mechanisms of cell-free lncRNA expression. We do want to point out that, as suggested by Raposo et al. [26], extracellular vesicles, such as exosomes, microvesicles and apoptotic bodies, can contribute to the remarkable stability of cell-free lncRNAs. We will design further studies to analyze the origin and 
protection mechanisms of cell-free lncRNAs in urine samples. Also, our study represented a single-center research. Larger number of independent cohorts from multi-centers are needed to validate our current findings. Finally, we will continue to improve the specificity of the constructed urinary lncRNA panel for bladder cancer diagnosis, especially for early-stage patients. We will also need to evaluate the discriminatory power of our lncRNA panel between bladder cancer patients and other patients with non-cancerous diseases (e.g., urinary tract infection).

In summary, we concluded that a panel of four lncRNA biomarkers could be used to differentiate bladder cancer from urocystitis, with high sensitivity and high specificity. This pave the way for further predictive study followed by pivotal clinical validation.

\section{Abbreviations}

IncRNA: long non-coding RNA; ROC: receiver operating characteristic; AUC: area under curve; NMIBC: non-muscle invasive bladder cancer; UCA1: Urothelial cancer associated 1; RUA: reactive urothelial atypia.

\section{Acknowledgements}

This work was supported by the National Natural Science Foundation of China (Grant No. 81572534), Natural Science Foundation of Shandong (Grant No. ZR2016HM32), Shandong Medical and Health Science and Technology Development Program (Grant No. 2016WSB01033), and Shandong Key Research and Development Plan (Grant No. 2018GSF118189).

\section{Competing Interests}

The authors have declared that no competing interest exists.

\section{References}

1. Lodewijk I, Dueñas M, Rubio C, Munera-Maravilla E, Segovia C, Bernardini A, et al. Liquid biopsy biomarkers in bladder cancer: A current need for patient diagnosis and monitoring. International journal of molecular sciences. 2018; 19: 2514 .

2. Bray F, Ferlay J, Soerjomataram I, Siegel RL, Torre LA, Jemal A. Global cancer statistics 2018: GLOBOCAN estimates of incidence and mortality worldwide for 36 cancers in 185 countries. CA: A Cancer Journal for Clinicians. 2018; 68: 394-424

3. Ferlay J, Soerjomataram I, Dikshit R, Eser S, Mathers C, Rebelo M, et al. Cancer incidence and mortality worldwide: Sources, methods and major patterns in GLOBOCAN 2012. International Journal of Cancer. 2015; 136: E359-E86.

4. Schned AR, Andrew AS, Marsit CJ, Kelsey KT, Zens MS, Karagas MR. Histological classification and stage of newly diagnosed bladder cancer in a population-based study from the Northeastern United States. Scandinavian journal of urology and nephrology. 2008; 42: 237-42.

5. Ye F, Wang L, Castillo-Martin M, McBride R, Galsky MD, Zhu J, et al. Biomarkers for bladder cancer management: present and future. American journal of clinical and experimental urology. 2014; $2: 1-14$

6. Babjuk M, Böhle A, Burger M, Capoun O, Cohen D, Compérat EM, et al. EAU guidelines on non-muscle-invasive urothelial carcinoma of the bladder: Update 2016. European Urology. 2017; 71: 447-61.
7. Kirkali Z, Chan T, Manoharan M, Algaba F, Busch C, Cheng L, et al. Bladder cancer: Epidemiology, staging and grading, and diagnosis. Urology. 2005; 66: 4-34.

8. Witzke KE, Großerueschkamp F, Jütte $\mathrm{H}$, Horn $\mathrm{M}$, Roghmann F, von Landenberg $\mathrm{N}$, et al. Integrated Fourier transform infrared imaging and proteomics for identification of a candidate histochemical biomarker in bladder cancer. The American Journal of Pathology. 2019; 189: 619-31.

9. Duquesne I, Weisbach L, Aziz A, Kluth LA, Xylinas E, Urology YAUUCGotEAo. The contemporary role and impact of urine-based biomarkers in bladder cancer. Translational andrology and urology. 2017; 6: 1031-42.

10. Du L, Duan W, Jiang X, Zhao L, Li J, Wang R, et al. Cell-free IncRNA expression signatures in urine serve as novel non-invasive biomarkers for diagnosis and recurrence prediction of bladder cancer. Journal of Cellular and Molecular Medicine. 2018; 22: 2838-45.

11. Chen M, Li J, Zhuang C, Cai Z. Increased lncRNA ABHD11-AS1 represses the malignant phenotypes of bladder cancer. Oncotarget. 2017; 8: 28176-86.

12. Yazarlou F, Modarressi MH, Mowla SJ, Oskooei VK, Motevaseli E, Tooli LF, et al. Urinary exosomal expression of long non-coding RNAs as diagnostic marker in bladder cancer. Cancer management and research. 2018; 10: 6357-65.

13. Wang X, Zhang Z, Wang H, Cai J, Xu Q, Li M, et al. Rapid identification of UCA1 as a very sensitive and specific unique marker for human bladder carcinoma. Clinical Cancer Research. 2006; 12: 4851-8.

14. Berrondo C, Flax J, Kucherov V, Siebert A, Osinski T, Rosenberg A, et al. Expression of the long non-coding RNA HOTAIR correlates with disease progression in bladder cancer and Is contained in bladder cancer patient urinary exosomes. PLOS ONE. 2016; 11: e0147236.

15. Luo $H$, Zhao $X$, Wan $X$, Huang $S$, Wu D. Gene microarray analysis of the lncRNA expression profile in human urothelial carcinoma of the bladder. International journal of clinical and experimental medicine. 2014; 7: 1244-54.

16. Huang M, Zhong Z, Lv M, Shu J, Tian Q, Chen J. Comprehensive analysis of differentially expressed profiles of lncRNAs and circRNAs with associated co-expression and ceRNA networks in bladder carcinoma. Oncotarget. 2016; 7: 47186-200.

17. Han Y, Liu Y, Nie L, Gui Y, Cai Z. Inducing cell proliferation inhibition, apoptosis, and motility reduction by silencing long noncoding ribonucleic acid metastasis-associated lung adenocarcinoma transcript 1 in urothelial carcinoma of the bladder. Urology. 2013; 81: 209.e1-.e7.

18. Li C, Cui Y, Liu L, Ren W, Li Q, Zhou X, et al. High expression of long noncoding RNA MALAT1 indicates a poor prognosis and promotes clinical progression and metastasis in bladder cancer. Clinical Genitourinary Cancer. 2017; 15: 570-6.

19. Seitz AK, Christensen LL, Christensen E, Faarkrog K, Ostenfeld MS, Hedegaard J, et al. Profiling of long non-coding RNAs identifies LINC00958 and LINC01296 as candidate oncogenes in bladder cancer. Scientific reports. 2017; 7: 395-

20. Wieczorek E, Reszka E. mRNA, microRNA and lncRNA as novel bladder tumor markers. Clinica Chimica Acta. 2018; 477: 141-53.

21. Wang F, Li X, Xie X, Zhao L, Chen W. UCA1, a non-protein-coding RNA up-regulated in bladder carcinoma and embryo, influencing cell growth and promoting invasion. FEBS Letters. 2008; 582: 1919-27.

22. Leiblich A. Recent developments in the search for urinary biomarkers in bladder cancer. Current urology reports. 2017; 18: 100-.

23. Wang $\mathrm{KC}$, Chang HY. Molecular mechanisms of long noncoding RNAs. Molecular cell. 2011; 43: 904-14.

24. Ishiba T, Hoffmann AC, Usher J, Elshimali Y, Sturdevant T, Dang M, et al. Frequencies and expression levels of programmed death ligand 1 (PD-L1) in circulating tumor RNA (ctRNA) in various cancer types. Biochemical and Biophysical Research Communications. 2018; 500: 621-5.

25. [Internet] MATLAB R2014b. Fitctree: Fit binary decision tree for multiclass classification. https://www.mathworks.com/help/stats/fitctree.html.

26. Raposo G, Stoorvogel W. Extracellular vesicles: Exosomes, microvesicles, and friends. The Journal of Cell Biology. 2013; 200: 373-83. 